\title{
Global transcriptome analysis of Bacillus cereus ATCC 14579 in response to silver nitrate stress
}

\author{
Malli Mohan Ganesh Babu', Jayavel Sridhar ${ }^{2}$ and Paramasamy Gunasekaran ${ }^{1,2^{*}}$
}

\begin{abstract}
Silver nanoparticles (AgNPs) were synthesized using Bacillus cereus strains. Earlier, we had synthesized monodispersive crystalline silver nanoparticles using B. cereus PGN1 and ATCC14579 strains. These strains have showed high level of resistance to silver nitrate $(1 \mathrm{mM})$ but their global transcriptomic response has not been studied earlier. In this study, we investigated the cellular and metabolic response of B. cereus ATCC14579 treated with $1 \mathrm{mM}$ silver nitrate for $30 \& 60 \mathrm{~min}$. Global expression profiling using genomic DNA microarray indicated that $10 \%(n=524)$ of the total genes $(n=5234)$ represented on the microarray were up-regulated in the cells treated with silver nitrate. The majority of genes encoding for chaperones (GroEL), nutrient transporters, DNA replication, membrane proteins, etc. were up-regulated. A substantial number of the genes encoding chemotaxis and flagellar proteins were observed to be down-regulated. Motility assay of the silver nitrate treated cells revealed reduction in their chemotactic activity compared to the control cells. In addition, 14 distinct transcripts overexpressed from the 'empty' intergenic regions were also identified and proposed as stress-responsive non-coding small RNAs.
\end{abstract}

Keywords: silver nitrate stress, silver nanoparticles, transcriptomics, Bacillus cereus, sRNA

\section{Background}

Metal nanoparticles exhibit unique electronic, magnetic, catalytic and optical properties that are different from those of bulk metals. Nanoparticles are synthesized using several physical and chemical methods such as laser irradiation, micelle, sol-gel method, hydrothermal and pyrolysis. Attempts are being made to develop nontoxic and environmental friendly methods for the production of metal nanoparticles using biological systems. The use of bacteria, fungi and yeast for the synthesis of metallic nanoparticles is rapidly gaining importance due to the success of microbial production of nanometals [1]. Heavy metals are essential as trace elements and they are found in high concentrations in marine environments, industrial effluents including mining and electroplating industries. Untreated effluents from these industries have an adverse impact on the environment.

Metal ions play important roles in microbial metabolism. Some metal ions are essential as cofactor in the metabolic reactions, others are oxidized or reduced to

\footnotetext{
* Correspondence: gunagenomics@gmail.com

'Department of Genetics, Centre for Excellence in Genomic Sciences, School of Biological Sciences, Madurai Kamaraj University, Madurai - 625 021, Tamil Nadu, India

Full list of author information is available at the end of the article
}

derive metabolic energy, while heavy metal ions such as $\mathrm{Ag}^{+}, \mathrm{Cd}^{2+}, \mathrm{Hg}^{2+}, \mathrm{Co}^{2+}, \mathrm{Cu}^{2+}, \mathrm{Ni}^{2+}, \mathrm{Zn}^{2+}$ cause toxic effects. To counter the toxic effects, microorganisms have evolved adaptive mechanisms to survive under metal ionic stress [2]. Bioremediation approach is getting more attention because of its economical and environmental friendly aspects. Metal contaminated industrial sites are bioremediated by stimulating indigenous microbial communities. Bacteria belonging to different genera such as Bacillus, Pseudomonas, Escherichia and Desulfovibrio have been shown to accumulate and reduce various heavy metals [3-5]. Ionic silver $\left(\mathrm{Ag}^{+}\right)$is known to be effective against wide range of microorganisms and has been traditionally used in therapeutics [6]. Basically, silver ions are charged atoms $\left(\mathrm{Ag}^{+}\right)$, whereas silver nanoparticles are zerovalent crystals of nanosize $(\mathrm{nm})$. The crystallized nanoparticles have been used as a source of $\mathrm{Ag}^{+}$ions in many commercial products, such as food packaging, odour resistant textiles, household appliances and medical devices. Despite growing concerns, little is known about the potential impacts of silver nanoparticles on human health and environment. Microbial resistance to silver is most likely to occur in environments where silver is routinely used; for example, burns units in

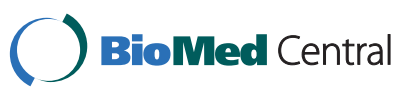


hospitals, catheters (silver-coated) and dental setting (amalgams contain 35\% silver). In spite of the fact that silver is known to exhibit bactericidal effect, its impact on the transcriptome and cellular physiology have not been studied [7-9].

Microorganisms have evolved adaptive mechanisms to face the challenges under silver ionic stress condition. $B$. cereus efficiently precipitates silver as discrete colloidal aggregates at the cell surface and occasionally in the cytoplasm, thus the organism has the ability to reduce $89 \%$ of the total $\mathrm{Ag}^{+}$and remove from the solution [10]. Similarly, B. licheniformis [11,12], B. cereus PGN1 [13], $B$. subtilis [14] were shown to accumulate silver nanoparticles with well defined size and shape, within the cytoplasm. Inside the cell, the toxic effects of heavy metals include nonspecific intracellular complexation with particularly vulnerable thiol groups. Previous studies reported that several heavy metals were toxic to cellular processes. In Gram-negative bacteria, heavy metal ions can bind to glutathione and the resulting products tend to react with molecular oxygen to form oxidized bis-glutathione, releasing the metal cation and hydrogen peroxide. Some metal ions structurally mimic physiologically important molecules. Some metals are reduced intracellularly by both enzymatic and non-enzymatic reactions. This process may inadvertently cause damage to many cellular components, including DNA and proteins. In addition, metal stress is associated with oxidase activity, biofilm formation, motility, oxidative stress or sulphur assimilation in various microorganisms $[12,15]$. However, the response exhibited by $B$. cereus at transcript level under silver ionic stress has not yet been studied.

The transcriptional response of Bacillus spp. to environmental perturbations can be large and complex, involving multiple transcription factors and their regulons. DNA microarrays of Bacillus spp. were already employed to study the global response under acid/base [16], peroxide [17], salt [18,19], organic/inorganic acid shocks [20], metal ions [21], superoxide radicals [22] and bile salts [23] stress conditions. Previously, some effector proteins in B. subtilis against multiple metal ion stresses were identified using DNA microarrays, but they were not studied for the global response against the metal ion stress. The availability of complete genome sequence of $B$. cereus ATCC14579 [NC_004722] [24] facilitates to design genome arrays which could be used for the analysis of global transcriptome in response to different stress conditions.

Recent studies have identified non-coding small RNAs (sRNAS) to play vital role in response to a variety of stress conditions. But very few small RNAs were reported in B. cereus ATCC14579 [25]. To search for additional sRNAs expressing in response to silver metal stress, we have included those 900 'empty' intergenic regions in the genomic microarray to detect transcripts arising from 'empty' intergenic regions of B. cereus. In this study, we performed DNA microarray for genome-wide transcriptional analysis of B. cereus ATCC 14579 in response to silver nitrate.

\section{Results and Discussion}

\section{Effect of silver nitrate on the viability of $B$. cereus}

The effect of silver nitrate induced stress on the growth of $B$. cereus ATCC14579 was studied by challenging the culture with silver nitrate. Figure 1 shows the viability of $B$. cereus upon treatment with $1 \mathrm{mM}$ silver nitrate. Exposure to silver nitrate decreased viability of the cells. Within $120 \mathrm{~min}$ exposure to silver nitrate, the viable cell number was decreased by two log scale i.e. from $10^{8}$ to $10^{6} \mathrm{cfu} / \mathrm{ml}$. These results suggested that silver nitrate treatment significantly affected the cell viability and growth of $B$. cereus ATCC14579.

\section{Characteristics of silver nanoparticles formed in B. cereus}

Scanning electron microscopy (SEM) analysis of the cells treated for $60 \mathrm{~min}$ with silver nitrate revealed the presence of silver nanoparticles within the cells (Figure 2A-C). Energy dispersive X-ray microanalysis (EDX) was done for qualitative analysis of the thin sections from the selected preparations. Elemental analysis of the silver nanoparticles was performed using EDX in SEM. The EDX spectrum of the silver nanoparticles synthesized by $B$. cereus is shown in Figure 2D. The vertical axis displays the number of $x$ ray counts whilst the horizontal axis displays energy (keV). The peaks between $3.00-3.40 \mathrm{keV}$ correspond to the binding energy of $\mathrm{Ag}_{\mathrm{La}}, \mathrm{Ag}_{\mathrm{Lb}}$ and $\mathrm{Ag}_{\mathrm{Lb} 2}$ with $~ 50-60$ counts, while the peaks near binding energies of $0.3 \mathrm{keV}$ and $0.52 \mathrm{keV}$ belongs to carbon and oxygen respectively. The carbon and oxygen peaks in the EDX analyses can be attributed to the surrounding residual material and/or the carbon tape used for SEM grid preparation. Throughout, the scanning range of binding energies, some peaks belonging to $\mathrm{Na}, \mathrm{Cl}$ and $\mathrm{P}$ were also detected $[26,27]$. SEM with EDX analysis of the colloids in the cell pellet indicated the presence of silver material in nano size diameters bound within the cell wall of the bacteria. These particles are in the monodispersed size range between 4- $5 \mathrm{~nm}$ and spherical shape, which is comparable with silver nanoparticles synthesised by other bacteria [13].

\section{Microarray experiments and their efficacy}

Transcriptome analysis was carried out with microarray to study the effect of silver nitrate stress response on the global gene expression in B. cereus. These experiments were conducted with a custom-designed $8 \times 15 \mathrm{~K}$ DNA microarray consisting of oligo probes for coding DNA sequences (CDS's) and intergenic regions (IGR's). The signal intensities obtained from the labelled cRNA 


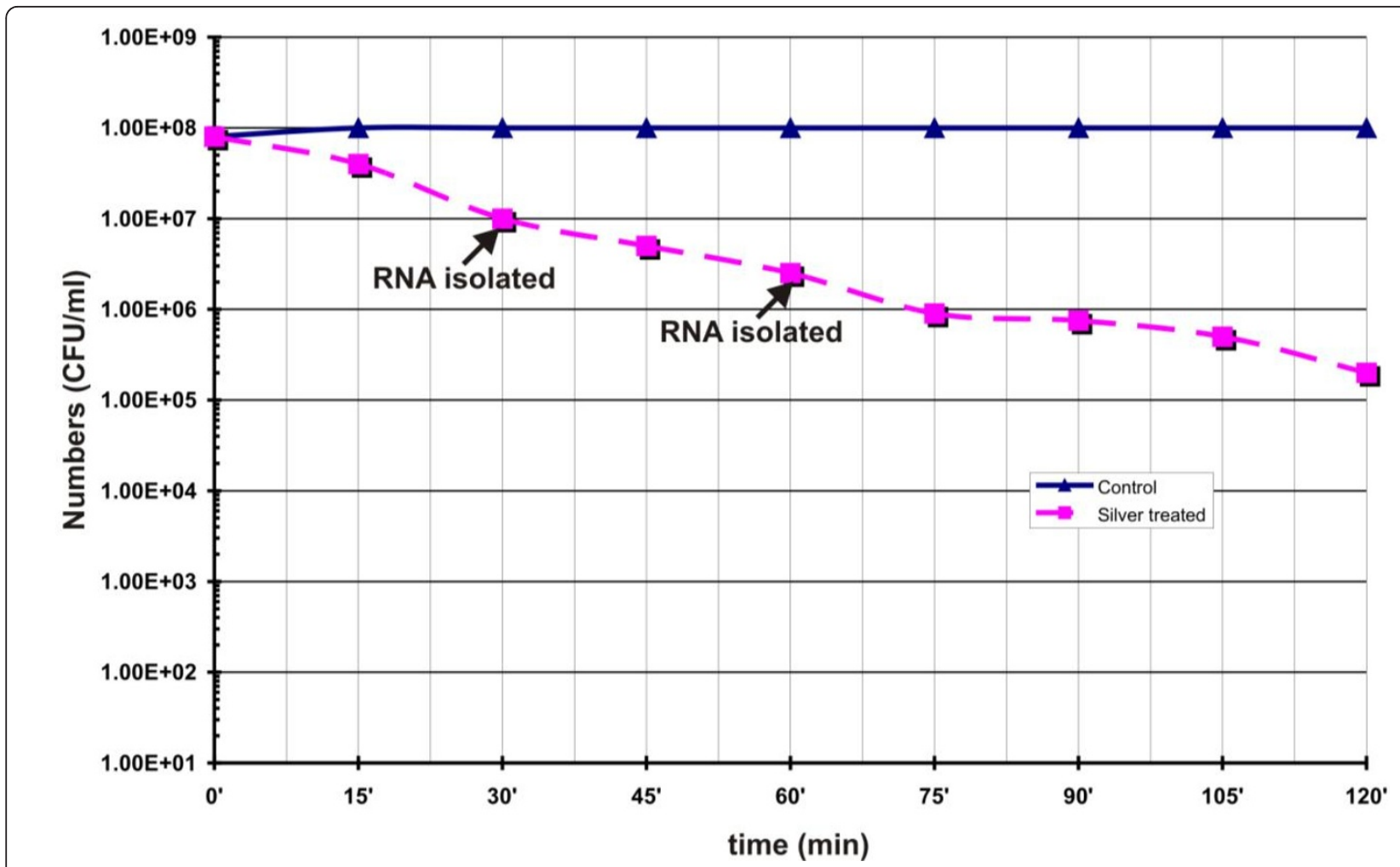

Figure 1 Survival of $B$. cereus cells during silver nitrate induced stress condition. Logarithmically grown cells were treated with and without $1 \mathrm{mM} \mathrm{AgNO}_{3}$. At intervals samples were withdrawn, suitably diluted and plated on LB agar plate without silver nitrate and incubated overnight at $37^{\circ} \mathrm{C}$. The colony forming units (CFU) were determined and plotted against time.

of the control cells $(30 \mathrm{~min})$ were presented in scatter plot to study the efficacy of the microarray experiments (Figure 3A). Virtually, majority of the spots lie on or close to the $45^{\circ}$ line suggesting no difference in gene expression between the biological duplicates. However, some of the genes showed low-intensity signals suggesting a high standard deviation because of background signal (i.e., intensities less than 100 arbitrary units or twice the detection limit are considered as not significantly expressed).

The scatter plot of signal intensities obtained from the cells grown with and without $1 \mathrm{mM} \mathrm{AgNO}$ revealed a clear difference in gene expression profile (Figure 3B). The genes that are up-regulated during silver nitrate stress condition showed signal intensity with at least one fold increase (shown by the upper and lower diagonal lines in Figure 3B). There was also more than a fivefold difference (lower or higher) in signal intensity as indicated by the diagonal lines. The hybridization signal intensity obtained from the control cells at 30 and 60 min (1a and 2a) showed majority of ORFs lying close to the diagonal and few others at the low intensity (Figure 3C). These hybridizations results suggested that over all precision analysis of the microarray using various statistical parameters is of greater accuracy [28].
Response to silver nitrate stress at transcript level

Genes showing differential expression in the cells exposed with silver nitrate at 30 and 60 min was compared with the controls. Expression of genes involved in basic cellular processes was classified based on the Clusters of Orthologous Groups (COG). Most of the genes that showed down-regulation during silver nitrate stress conditions were identified to fall under the COG functional classes of cell motility $(\mathrm{N})$, translation $(\mathrm{J})$ and hypothetical proteins. Interestingly, transcripts encoding proteins of transport and metabolism (P) (such as inorganic ion, amino acids, carbohydrate, synthesis of drug/antibiotics and oligopeptide), transcription (K), DNA replication/recombination/ repair $(\mathrm{L})$, transcriptional regulators and cell envelope biogenesis/membrane $(\mathrm{M})$ were found to be up-regulated in cells exposed to $\mathrm{AgNO}_{3}$ stress (Figure 4A-B). Generally, genes encoding transporters and membrane proteins (e.g. efflux proteins, drug resistance transporters, transcriptional regulators) were found to be up-regulated upon metal ionic stress conditions. These results also confirmed that the induction of osmoprotectant transporters during exposure to silver stress condition. In the genome of $B$. cereus, genes encoding various osmoprotectant transporters were identified. The osmoprotectant gene encoding a proline/betaine transporter belonging to the major 

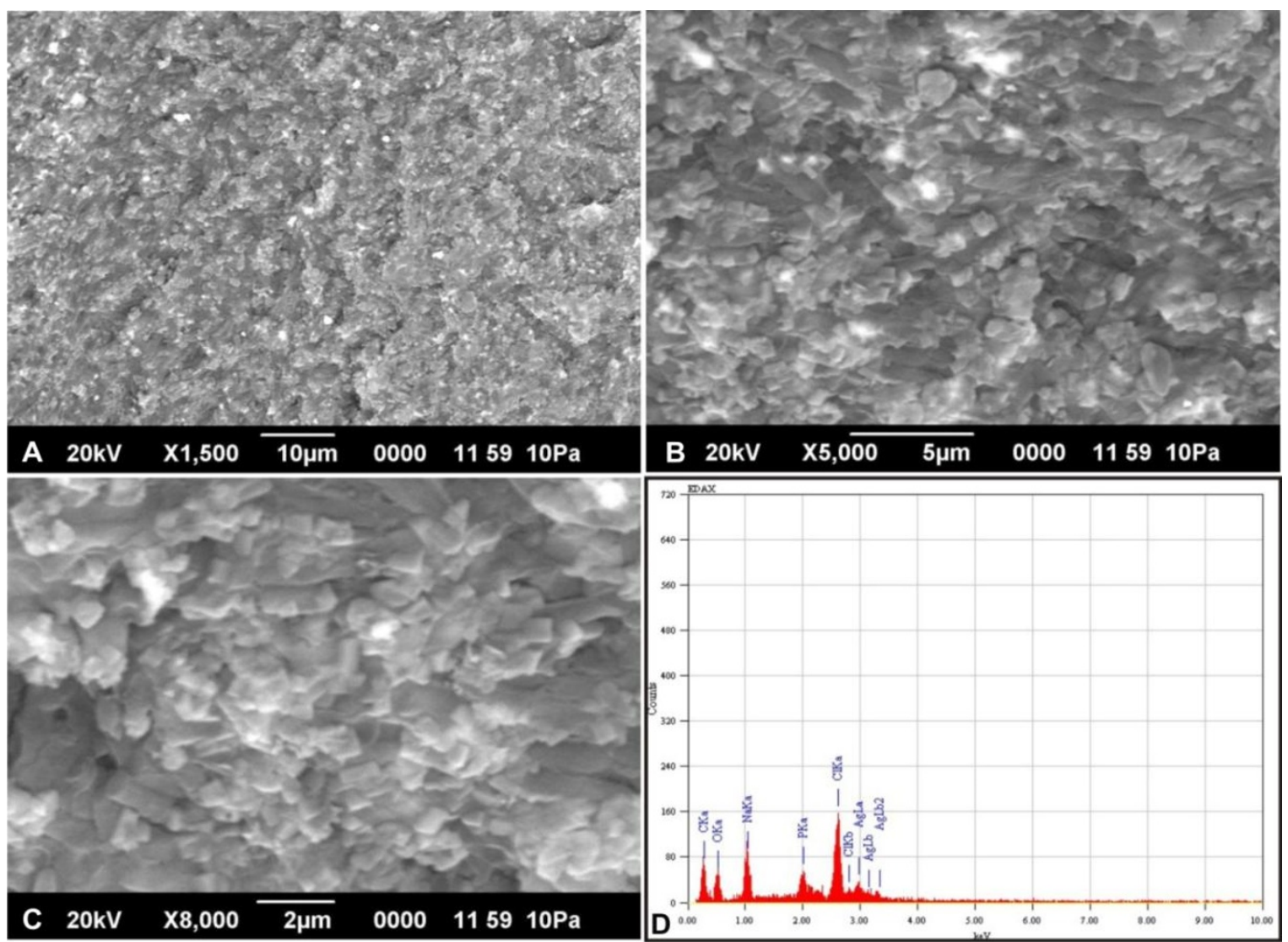

Figure 2 SEM with EDX analysis of silver nanoparticles synthesized by B. cereus ATCC 14579. Scanning Electron microscopy image (A - C) represents silver nanoparticles synthesised from $B$. cereus ATCC 14579 incubated at $37^{\circ} \mathrm{C}$ for $1 \mathrm{~h}$. Figure $2 \mathrm{D}$ represents the Energy-Dispersive $\mathrm{X}$-ray microanalysis of silver nanoparticles.

facilitator transporter family and a gene encoding a proton-dependent di-, tri- and oligopeptide transporter were among the highly induced genes upon exposure to silver stress. In addition, the genes encoding $\mathrm{ABC}$ transporters OpuA [BC2791] and OpuB/OpuC [BC2232] were found to be induced during silver nitrate treatment. Furthermore, the up-regulation of zinc-transporting ATPase [BC0596], cationic $\mathrm{Na}^{+} / \mathrm{H}^{+}$antiporters [BC0373 and $\mathrm{BC} 0838$ ] and copper importing ATPase [BC3730] a part of cop system were induced upon silver ionic stress. Another $\mathrm{Na}^{+} / \mathrm{H}^{+}$antiporter encoded by [BC1612] have been reported to be induced under salt stress but it was observed to be stably expressed upon silver stress. Higher level of expression P-type ATPases in B. cereus ATCC14579, a versatile group of ion pumps, has suggested that it contributes to the metal homeostasis in response to silver stress [18]. The cop system is a general metal response system that is readily inducible at lower to higher levels of metal stress caused by $\mathrm{Cu}(\mathrm{II})$ and $\mathrm{Ag}(\mathrm{I})$. Previous report on transcriptional activator like CueR, an Mer R-like was found to respond to $\mathrm{Cu}(\mathrm{II})$ and it was also activated by $\operatorname{Ag}(\mathrm{I})[19,29]$.
Heat shock proteins (GroEL, GroES, DnaJ and DnaK) are generally induced in microorganisms under various stress conditions [30-32]. But in our study, GroEL [BC0295] alone was up-regulated at the early stages of silver ionic stress. Generally, oxidative stress response genes are involved in response to metal ionic stresses in bacteria. Both, the vegetative catalase-KatA [BC1155] and $\sigma^{\mathrm{B}}$-dependent catalase-KatE [BC0863] genes were commonly known to respond to oxidative stresses, but in our study, KatE [BC0863] alone was induced upon silver ionic stress and presumed to have essential role in the survival of the cell. In addition, NAD and NADH dependent enzymes especially nitrate reductase [BC2118] and nitroreductase [BC3024] were found to be up-regulated during silver nitrate treatment. The involvement of nitrate reductase in the production of silver nanoparticles has been previously demonstrated $[12,33]$.

The important transcriptional activators of stress sigma factor $\left(\sigma^{\mathrm{B}}\right), r s b Y$ [BC1006] and $r s b V$ [BC1004] were found to be up-regulated during 30 and 60 min exposure to silver nitrate. The up-regulation of anti-sigma factor antagonist $(r s b Y)$ indicates the activation of $\sigma^{\mathrm{B}}$ induced 

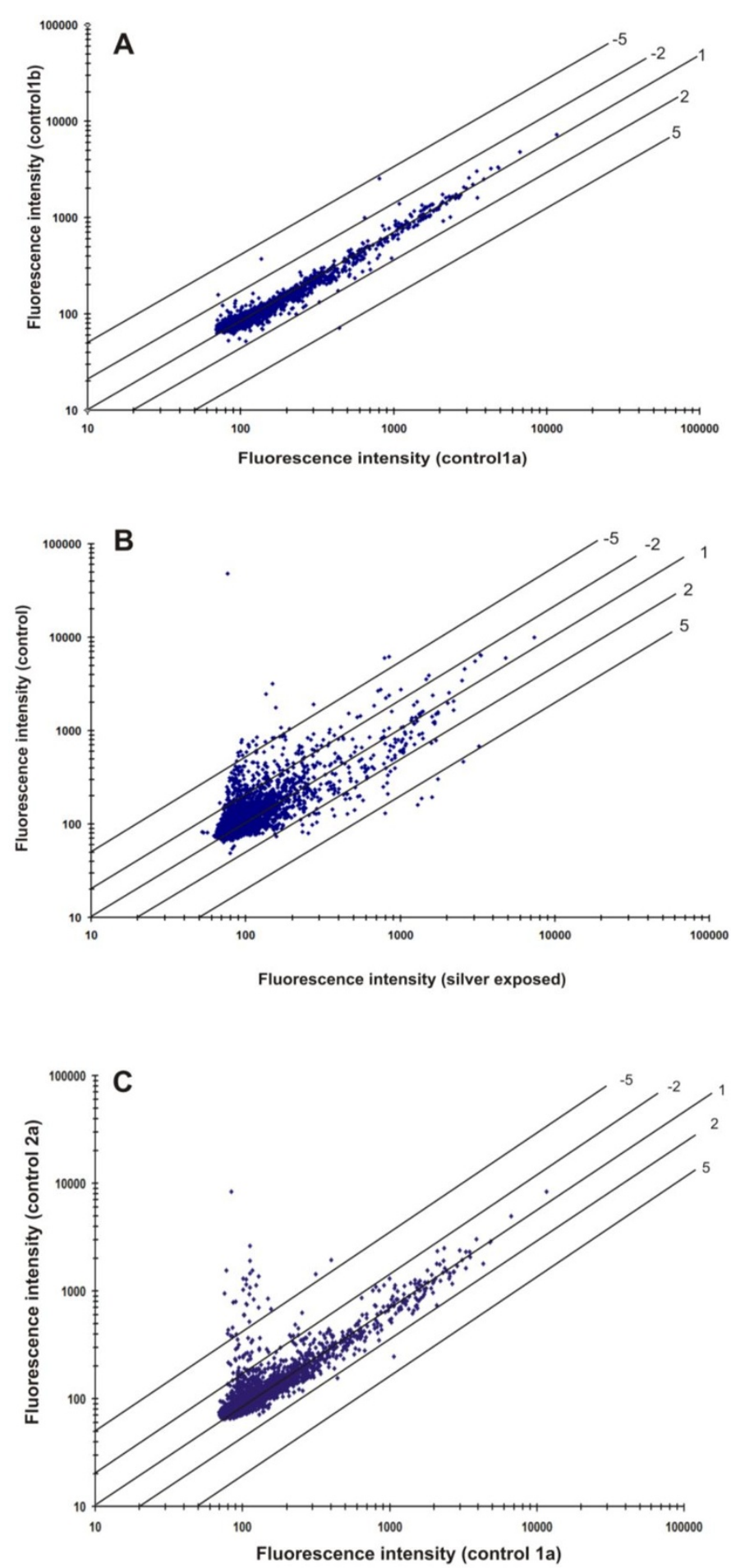

Figure 3 Scatter plots of normalized spot fluorescence intensities (arbitrary units) from DNA microarrays. (A) Spot intensities of array hybridizations with two different control samples in duplicates from the B. cereus ATCC 14579 (control 1a versus control 1b). (B) Comparison of spot intensities of array hybridizations from control samples (control 1a and $\mathrm{AgNO}_{3}$ treated sample test 1a). (C) Spot intensities of the array hybridized from control samples at $30 \mathrm{~min}$ (control 1a) and $60 \mathrm{~min}$ (control 2a). 


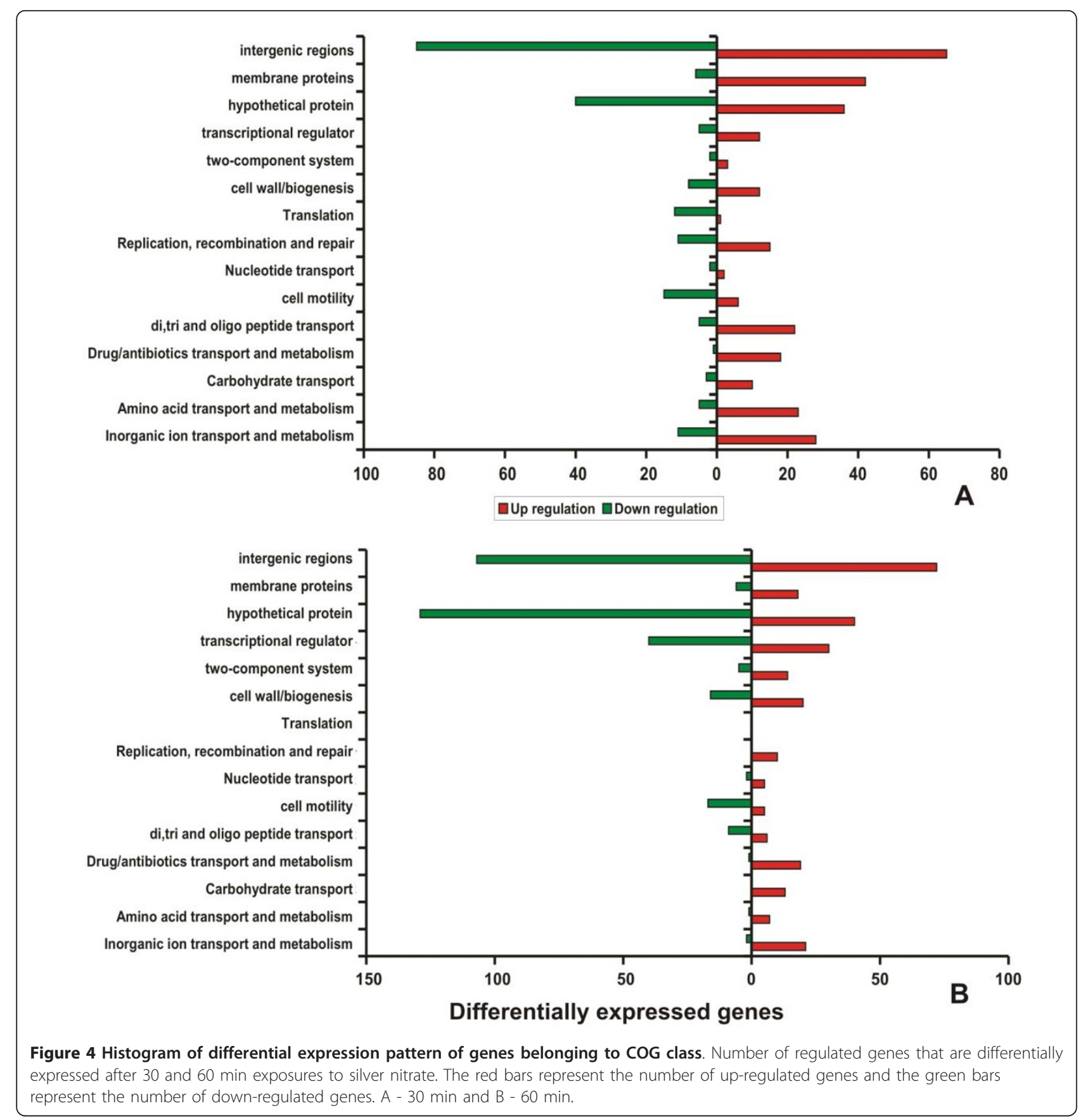

global stress response. Most of these induced genes are under the control of alternative sigma factor $\left(\sigma^{\mathrm{B}}\right)$ in response to variety of stress conditions acting via partner switching mechanisms in Gram-positive bacteria $[23,30,34]$. Interestingly, silver ion stress is known to induce metabolic pathways associated with amino acid metabolism especially arginine metabolism [21]. The present study has confirmed the over expression of arginine utilization protein [BC0473]. The S-adenosyl methionine -dependent methyl transferase [BC2891] and S-adenosyl homocysteine nucleosidase [BC2503], which are essential for the cellular detoxification of metals were also upregulated during 30 and $60 \mathrm{~min}$ exposure to silver nitrate $[35,36]$. Summary of gene expression pattern of B. cereus ATCC 14579 in response to silver nitrate stress is listed in Additional file 1.

Several genes associated with cell motility are located in a $\sim 45 \mathrm{~kb}$ region ranging from locus [BC1625] to locus 
[BC1671] in the B. cereus ATCC14579 genome [23,24]. Most of the flagellar and chemotactic genes were down regulated at transcript level upon silver stress are shown in Figure 5. Most of the chemotaxis related genes (CheY, CheA and $\mathrm{CheV}$ ), flagellar biosynthetic genes ( $f l i \mathrm{P}$ and $f l i \mathrm{Q})$, hook-associated genes (flgL, flgE and $f l i \mathrm{E}$ ), basal body rod genes ( $f l g \mathrm{~B}$ and $f l g G$ ) and motor switch genes (fliG and $f l i \mathrm{~N})]$ were down-regulated after silver nitrate treatment. Interestingly, there was no change in the expression level of flagellar motor switch ( $f l i \mathrm{R})$, a component of type III secretion system, suggesting that it may not have any role in the ionic stress response. In contrast, most of the flagellar genes exhibited dramatic changes in their expression within 30 min after silver nitrate treatment. But, few of them were down regulated for continued exposure to silver nitrate for $60 \mathrm{~min}$ (Figure 5). The genes encoding flagellar components such as basal ring, hook, hook filament junction and cap ( $f l i \mathrm{M}, f l g \mathrm{~K}, f l i \mathrm{D}, f l g \mathrm{E}, f l g \mathrm{D}$ and $f l g \mathrm{C}$ ) were induced in $B$. cereus during early stages of silver stress (30 min). The prolonged silver stress leads to the decreased level of expression of genes involved in biosynthesis of flagellar components. In addition, fliM gene is also involved in the generation of energy from transmembrane electrochemical ion gradients, in the form of $\mathrm{Na}^{+} / \mathrm{H}^{+}$antiporter (sodium proton motive force) into mechanical energy [37]. At an early stage of silver stress, the genes involved in ion gradients were induced while the prolonged stress repressed the motive force. Our microarray expression profiling study has shown strong evidence for suppression of motility and chemotaxis under silver nitrate stress. This result was supported by the differential expression of chemotaxis and motility related genes in $B$. cereus, $B$. subtilis and $H$. arsenicoxydans strains in response to various stresses $[18,23,38]$. Further, the chemotactic behaviour of silver stressed B. cereus cells were examined on swarming plates (Figure 6). These results suggested that prolonged exposure of silver nitrate delayed the cell motility.

\section{Identification of sRNAs in Intergenic regions}

Bacterial small and un-translated RNAs serve as crucial regulators of cellular physiology to environmental signals. These sRNAs act against the effectors either at transcription or translation levels. We used genome based microarrays to identify, the expression of candidate sRNAs predicted in the intergenic regions upon silver ionic

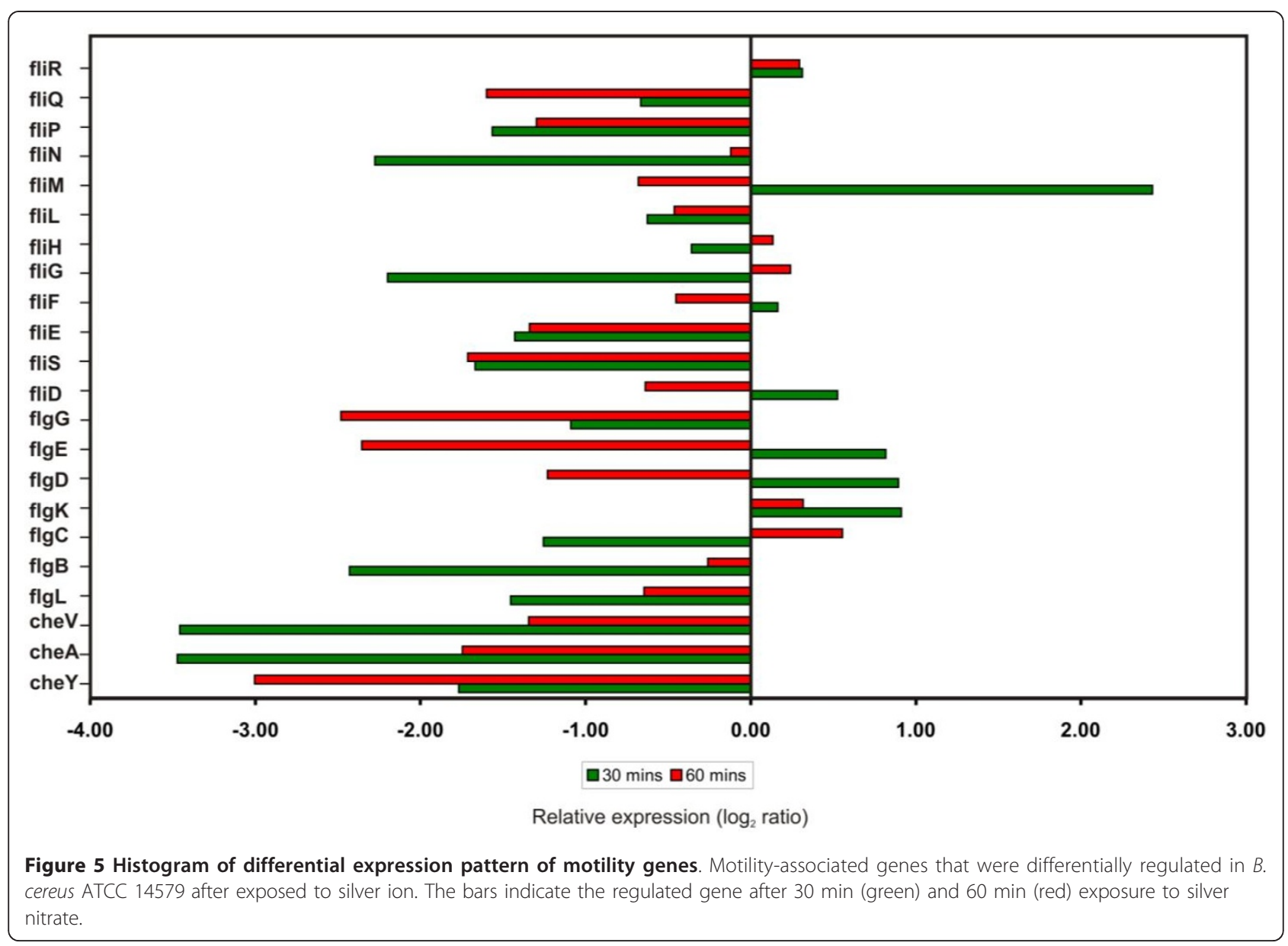



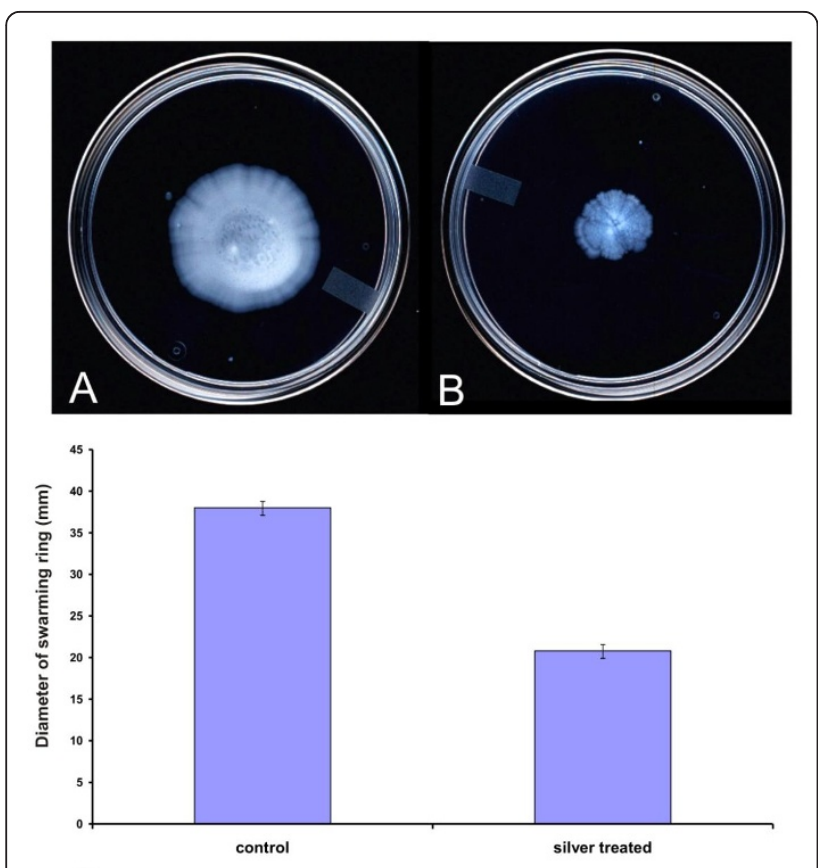

C

Figure 6 Influence of silver nitrate stress on motility of $B$. cereus. The B. cereus ATCC 14579 was inoculated onto swarming plates without (A) or with exposure to $1 \mathrm{mM}$ silver nitrate (B). After growth at $37^{\circ} \mathrm{C}$ overnight, plates were observed for swarming capability. (C) The level of motility at $12 \mathrm{~h}$ was evaluated as the diameter of the swarming ring in $\mathrm{mm}$. The results presented are the mean value of three independent experiments.

stress. An intergenic region is assumed to encode a sRNA and that can bind to its specific cognate probes which are complementary to each other. The distribution of sRNA and the possible transcripts from each and every 'empty' intergenic region was modelled from the genome of $B$. cereus shown in Figure 7 and 8. Possible intergenic transcripts as parallel transcriptional outputs from adjacent mRNAs in the same strand are shown in Figure 8A, B $\& 8 \mathrm{D}$. The transcripts expressing from the UTRs of the adjacent genes are shown in Figure $8 \mathrm{E} \& 8 \mathrm{G}$. The nonoverlapping intact sRNA located within the intergenic region which has diverged orientations with its upstream flanking gene is considered as 'True' sRNA (Figure 8C $\& 8 \mathrm{~F})$. The expression pattern of a given transcript was determined in accordance with the computer-assisted algorithm of the Agilent system. In this way, fourteen putative sRNAs collectively from $30 \mathrm{~min} \& 60 \mathrm{~min}$ conditions were detected according to their diverging orientations with their adjacent flanking genes. Interestingly, the expression of ten putative sRNAs (sRNA1 to sRNA10) were found under early period (30 $\mathrm{min})$ of exposure to $\mathrm{AgNO}_{3}$ stress while other four putative sRNAs (sRNA11 to sRNA14) were found after prolonged (60 min) exposure to $\mathrm{AgNO}_{3}$ stress (Table 1). We have compared the

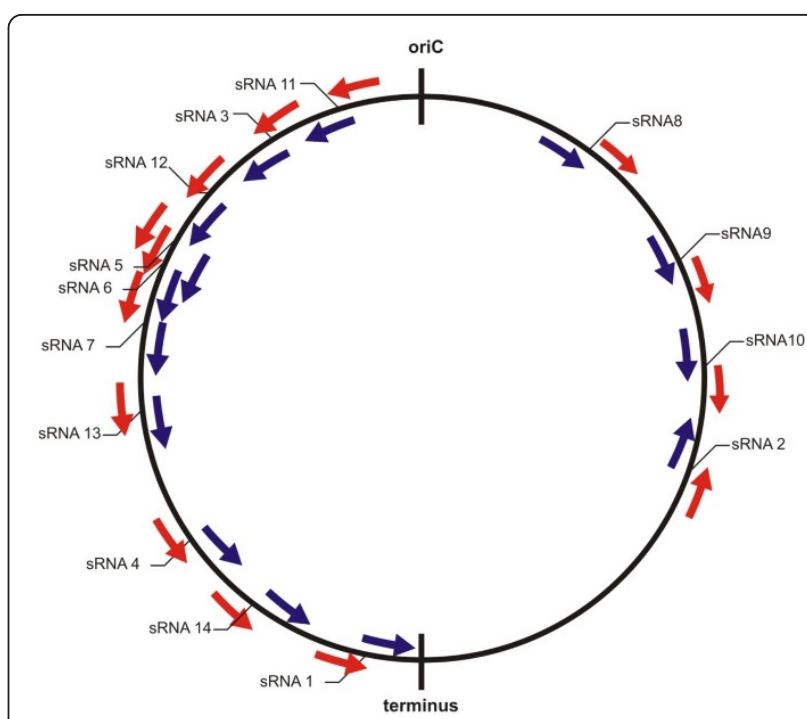

Figure 7 Distribution of the putative sRNA-encoding genes along the $\boldsymbol{B}$. cereus genome. The origin and terminus of replication are indicated. sRNA genes on the leading and lagging strands are coloured blue and red, respectively.

locations of the fourteen putative sRNAs with the computational sRNA predictions by sRNA scanner [39]. This observation has confirmed our initial prediction and detection of fourteen putative silver stress responsive sRNAs proposed from this study. These sRNA candidates are presumed to have regulatory roles under silver ionic stress conditions but needs further validations to understand their actual functions. Interestingly, the overexpression of the small RNA binding chaperone protein Hfq [BC1623] was found upon silver ionic stress. It clearly indicates the unknown regulatory cascade of the abundant small RNAs and Hfq protein which needs further validation.

\section{Conclusions}

Chemical and biological methods are used for the synthesis of silver nanoparticles with significantly novel structures, improved physicochemical and biological properties. Bacterial species of Bacillus, Pseudomonas and certain filamentous fungi are widely used for the biological synthesis of silver nanoparticles. Here, we have studied the transcriptomic and phenotypic responses of the B. cereus ATCC 14579 during the biosynthesis of silver nanoparticles. The bacteria can activate various cellular and metabolic adaptive mechanisms to reduce the toxicity and precipitate silver as nano-sized particles. Several microbes are reported to produce silver nanoparticles from the aqueous silver nitrate $(\sim 1 \mathrm{mM})$ and several proteins are expected to play vital role in the detoxification and precipitation of silver nanoparticles. The transcriptome analysis of B. cereus ATCC14579 exposed to 


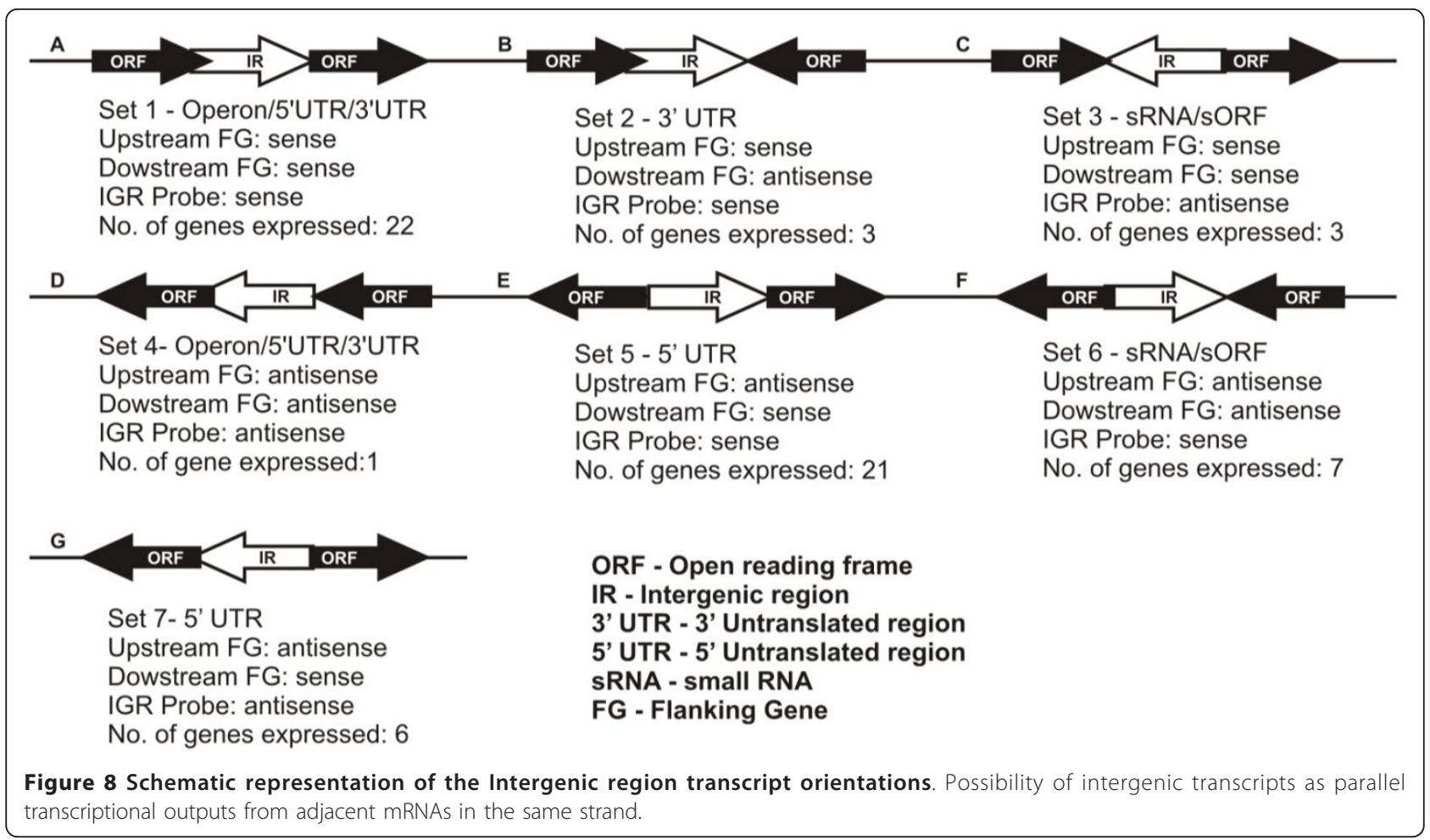

silver ionic stress was done using whole-genome DNA microarrays. Approximately $10 \%$ of the genes were upregulated but $20 \%$ of the genes were down regulated upon silver ionic stress. The SEM along with EDX analysis has revealed the accumulation of Ag nanoparticles in the cell-wall. In general, silver stress which has induced the expression of genes involved in the osmoprotection, transport elements, oxidative stress response and detoxification may have contributed to cross protection. Interestingly, silver ionic stress was observed to delay the cell motility. These characteristic phenotypic assessments can contribute to a better understanding of cellular stress adaptation strategies. Finally, fourteen 'putative' transcripts were found to be induced from the 'empty'

Table 1 Summary of RNA transcripts within intergenic regions of B. cereus ATCC14579 regulated in response to silver nitrate identified by genome based microarray

\begin{tabular}{|c|c|c|c|c|c|c|c|c|c|}
\hline \multirow[t]{2}{*}{ sRNA id } & \multirow[t]{2}{*}{ Start } & \multirow[t]{2}{*}{ End } & \multirow{2}{*}{$\begin{array}{l}\text { Length } \\
\text { (nucleotides) }\end{array}$} & \multirow[t]{2}{*}{ Flanking gene id } & \multirow[t]{2}{*}{ Strand* } & \multirow[t]{2}{*}{ Expression pattern } & \multirow[t]{2}{*}{ Relative copy numbers } & \multicolumn{2}{|c|}{ Primers } \\
\hline & & & & & & & & Start & End \\
\hline sRNA1 & 2840862 & 2840921 & $\sim 60$ & BC2880/BC2881 & $<><$ & $\downarrow \uparrow \downarrow$ & 54 & 2840862 & 2840921 \\
\hline sRNA2 & 1502573 & 1502638 & $\sim 66$ & BC1553/BC1554 & $<><$ & $\downarrow \uparrow \downarrow$ & 38 & 1502576 & 1502635 \\
\hline sRNA3 & 5024909 & 5024970 & $\sim 62$ & BC5122/BC5123 & $<><$ & $\downarrow \uparrow \downarrow$ & 36 & 5024910 & 5024969 \\
\hline sRNA4 & 3372000 & 3372189 & $\sim 190$ & BC3408/BC3409 & $<><$ & $\downarrow \uparrow \downarrow$ & 181 & 3372130 & 3372189 \\
\hline sRNA5 & 4261246 & 4261455 & $\sim 210$ & BC4317/BC4318 & $<><$ & $\downarrow \uparrow \downarrow$ & 41 & 4261395 & 4261454 \\
\hline sRNA6 & 4219221 & 4219336 & $\sim 116$ & BC4271/BC4272 & $<><$ & $\downarrow \uparrow \downarrow$ & 56 & 4219276 & 4219335 \\
\hline sRNA7 & 4040528 & 4040740 & $\sim 213$ & BC4070/BC4071 & $<><$ & $\downarrow \uparrow \downarrow$ & 169 & 4040679 & 4040738 \\
\hline sRNA8 & 525752 & 526136 & $\sim 385$ & BC0547/BC0548 & $><>$ & $\downarrow \uparrow \downarrow$ & 142 & 526073 & 526132 \\
\hline sRNA9 & 1106401 & 1106863 & $\sim 463$ & BC1124/BC1125 & $><>$ & $\downarrow \uparrow \downarrow$ & 44 & 1106797 & 1106856 \\
\hline sRNA10 & 1381925 & 1382221 & $\sim 297$ & BC1419/BC1420 & $><>$ & $\downarrow \uparrow \downarrow$ & 183 & 1382156 & 1382215 \\
\hline sRNA11 & 5372154 & 5372598 & $\sim 445$ & BC5447/BC5448 & $<><$ & $\downarrow \uparrow \downarrow$ & 42 & 5372540 & 5372599 \\
\hline sRNA12 & 4790423 & 4791589 & $\sim 1167$ & BC4864/BC4865 & $<><$ & $\downarrow \uparrow \downarrow$ & 19 & 4791428 & 4791487 \\
\hline sRNA13 & 3802805 & 3802907 & $\sim 103$ & BC3824/BC3825 & $<><$ & $\downarrow \uparrow \downarrow$ & 16 & 38028445 & 3802904 \\
\hline sRNA14 & 3121534 & 3121698 & $\sim 165$ & BC3151/BC3152 & $<><$ & $\downarrow \uparrow \downarrow$ & 12 & 3121639 & 3121698 \\
\hline
\end{tabular}

*The middle symbol represents the sRNA gene, while the flanking symbol indicates the orientation of the adjacent genes, respectively. Genes present on the strand given in the $B$. cereus genome database are indicated by $(>)$, and genes present on the complementary strand are indicated by $(<)$. 
intergenic regions upon silver nitrate stress and they are proposed as stress responsive putative sRNAs which could be studied in detail for their role in differential gene expressions.

\section{Materials and methods}

\section{Bacterial strain culture conditions and SEM analysis}

Gram-positive Bacillus cereus ATCC 14579 was used as a model system in this study. The strain was routinely grown in LB broth containing yeast extract $(10 \mathrm{~g} / \mathrm{L})$, peptone $(10 \mathrm{~g} / \mathrm{L})$ and $\mathrm{NaCl}(5 \mathrm{~g} / \mathrm{L})$ and $\mathrm{pH}-8.0)$ and incubated at $37^{\circ} \mathrm{C}$ with agitation $(200 \mathrm{rpm})$. The overnight cultures were diluted to 1:100 in $100 \mathrm{ml}$ pre-warmed LB broth and incubated at $37^{\circ} \mathrm{C}$, with shaking at $200 \mathrm{rpm}$ until the cells were growing exponentially. When an optical density at $600 \mathrm{~nm}\left(\mathrm{OD}_{600}\right)$ of $0.5-0.6$ was reached, decimal dilutions were prepared using $9 \mathrm{ml}$ of a peptone saline solution and plated on LB agar to determine the viable counts.

To study the effect of silver nitrate exposure, the exponentially grown cells were treated with $1 \mathrm{mM}$ silver nitrate and incubated at $37^{\circ} \mathrm{C}$. At intervals, aliquots of control and silver treated cultures were diluted and plated on LB agar plates. The plates were incubated for $24 \mathrm{~h}$ at $37^{\circ} \mathrm{C}$ and the viable cells were expressed as $\log _{10}$ colony forming units (CFU). The $\mathrm{AgNO}_{3}$ treated cultures and corresponding controls at two different time points (30 and $60 \mathrm{~min}$ ) were used for gene expression profiling. For each condition of microarray analysis, biological duplicates were prepared.

The accumulation of silver nanoparticles within the B. cereus cells after $\mathrm{AgNO}_{3}$ treatment for 60 min was recorded with a JEOL Model JSM - 6390LV and JEOL Model JED - 2300 operating at $1 \mathrm{pA}$ to $1 \mathrm{~mA}$ with a 3 , 8 and $15 \mathrm{~nm}$ resolution (JEOL Model JSM - 6390LV and JEOL Model JED - 2300, Tokyo, Japan). Samples were collected by centrifugation $(8000 \mathrm{rpm})$ for $10 \mathrm{~min}$, dried in oven at $60^{\circ} \mathrm{C}$ overnight. Approximately, $1 \mathrm{~g}$ of finely powdered sample was used for SEM-EDX analysis.

\section{Microarray design}

A sense and antisense oligonucleotide microarray slides complementary to the B. cereus ATCC14579 genome was custom-designed (obtained from Agilent technologies, USA) using the published DNA sequence [NC_004722] [24]. Antisense oligonucleotide sequences targeting the 'empty' intergenic regions were also designed on the customized genome array. Each oligonucleotide probe was 60 nucleotides in length and was specifically designed using eArray software from Agilent technologies http://earray. chem.agilent.com/earray. Totally, 15,000 probe sets were designed for Gene Expression profiling of $B$. cereus using $8 \times 15$ k Array AMADID: 23971. It targets the 5234 annotated CDS's, 900 'empty' intergenic regions located on the genome and CDS's encoded by the 21 plasmids.

\section{Total RNA isolation and CDNA preparation}

Bacterial RNA was isolated using the Qiagen RNeasy kit and on-column DNA digestion was carried out according to manufacturer's instructions (Qiagen, Hilden, Germany), additional DNA removal was done by with DNase I (Ambion, Austin, USA). To perform this, the RNA was precipitated and re-constituted in $85 \mu \mathrm{l}$ of nuclease-free water and then added with $10 \mu \mathrm{l}$ of $10 \times$ DNase I buffer and $5 \mu \mathrm{l}$ of $(1 \mathrm{U} / \mu \mathrm{l})$ DNase I. The reaction mixture was incubated at $37^{\circ} \mathrm{C}$ for $30 \mathrm{~min}$ and then chilled on ice. A second RNeasy column purification was performed. The RNA isolation protocol for Gram-positive bacteria was followed, in which $3 \mathrm{mg} / \mathrm{ml}$ of lysozyme was used to degrade the bacterial cell wall. The RNA quality, purity and integrity were determined using both NanoDropTM 1000 spectrophotometer (Thermo Scientific, Wilmington DE, USA) and RNA 6000 Nano Lab Chips with an Agilent 2100 Bioanalyzer (Agilent Technologies, Santa, CA, USA). Standard methods were used for cDNA synthesis, fragmentation and cyanine3 labelling as per the manufacturer's protocol (Genotypic technologies, Bangalore).

\section{CRNA preparation, microarray hybridization}

The synthesized cDNA was transcribed into cRNA using in vitro transcription kit (Agilent Technologies, CA, USA) and labelled with cyanine 3 labelled nucleotide according to manufacturer's protocol and purified with RNeasy Mini columns (Qiagen, Hilden, Germany). The quality of the labelled cRNA sample was verified by the total yield and specificity calculated based on NanoDrop ND-1000 spectrophotometer (Thermo Scientific, Wilmington DE, USA). Labelled cRNAs with specificity greater than 7 were considered as of high quality and taken for hybridization using the in situ hybridization kit plus (Agilent Technologies, Santa, CA, USA). Then, the arrays were incubated at $65^{\circ} \mathrm{C}$ for $16 \mathrm{~h}$ in Agilent's microarray hybridization chambers and the hybridized slides were washed according to the manufacturer's protocol.

\section{Image processing and Data analysis}

Arrays were scanned at $5 \mu \mathrm{m}$ resolution using the Agilent Microarray Scanner G Model G2565BA and images were saved as TIFF format. Data extractions from images were performed with the Feature Extraction software v 10.5.1 and GeneSpring GX version 11.0 software (Agilent Technologies, Santa, CA, USA). Normalization of the data was carried out by GeneSpring GX using the percentile shift normalization which is a global normalization, where the locations of all the spot intensities in an array are adjusted. This normalization takes each column in an experiment independently, and computes the $n^{\text {th }}$ percentile of the expression values for this array, across all spots (where $\mathrm{n}$ has a range from $0-100$ and $\mathrm{n}=75$ is the median). It subtracts this value from the expression value of 
each entity and normalized to specific samples. After normalization, controls were removed and replicates corresponding to the same genes within each slide were averaged, provided that there were at least two replicates left after the initial filtering procedure. Otherwise, the gene entry was removed. The average values were compared across slides. The model was evaluated on the basis of that $P$ values computed using a false discovery rate correction. Genes were considered regulated at a statistically significant level if they had a $P$ value below 0.05 and a ratio of \pm 1 cut-off relative to the reference cDNA. Several controls were employed to minimise the technical and biological variations and ensure the quality of the data: 1) each ORFs were present in duplicates in each array 2) array slides were prepared in duplicates for each experiments and 3) two independent RNA batches from each condition were used.

\section{Microarray data submission and accession numbers}

The microarray data derived from this study have been deposited in the National Center for Biotechnology Information (NCBI) Gene Expression Omnibus http://www. ncbi.nlm.nih.gov/geo and are accessible through GEO accession number - [GSE26043].

\section{Motility Assay}

Cells from exponentially grown cultures with and without $1 \mathrm{mM} \mathrm{AgNO}$ treatment for $60 \mathrm{~min}$ were recovered by centrifugation at $10,000 \times \mathrm{g}$ at $4^{\circ} \mathrm{C}$ for $1 \mathrm{~min}$. The sample volume of the silver stressed cells was adjusted to equivalent cell density and inoculated into LB-soft agar $\left(0.4 \%\right.$ agar) plates and incubated at $37^{\circ} \mathrm{C}$ overnight.

\section{Additional material}

Additional file 1: Supplemental information to manuscript Expression patters of genes responding to silver nitrate stress.

\begin{abstract}
Acknowledgements
Authors thank the University Grants Commission (UGC) New Delhi, Government of India for the financial support received through University with Potential for Excellence (UPE) scheme to Madurai Kamaraj University. Authors also thank UGC-Networking Resource Centre in Biological Sciences and Centre for Excellence in Genomic Sciences in School of Biological Sciences for support facilities.
\end{abstract}

\section{Author details}

${ }^{1}$ Department of Genetics, Centre for Excellence in Genomic Sciences, School of Biological Sciences, Madurai Kamaraj University, Madurai - 625 021, Tamil Nadu, India. ${ }^{2}$ UGC-Networking Resource Centre in Biological Sciences, School of Biological Sciences, Madurai Kamaraj University, Madurai - 625 021, Tamil Nadu, India.

\section{Authors' contributions}

MMGB has made substantial contributions to conception, design, acquisition, collection, analysis and interpretation of data and wrote the paper. JS carried out the computational analyses of sRNA. PG provided research support and involved in drafting and revising the manuscript critically for important intellectual content. All authors read and approved the final manuscript.

\section{Competing interests}

The authors declare that they have no competing interests.

Received: 26 July 2011 Accepted: 10 November 2011

Published: 10 November 2011

\section{References}

1. Mandal D, Bolander ME, Mukhopadhyay D, Sarkar G, Mukerjee P: The use of microorganisms for the formation of metal nanoparticles and their application. Appl Microbiol Biotechnol 2006, 69:485-492

2. Reith F, Etschmann B, Grosse C, Moors H, Benotmane MA, Moniseurs P, Grass G, Doonan C, Vogt S, Lai B, Martinez-Criado G, George GN, Nies DH, Mergeay M, Pring A, Southam G, Brugger J: Mechanisms of gold biomineralization in the bacterium Cupriavidus metallidurans. PNAS 2009, 106:17757-17762.

3. Shirdam R, Khanafari A, Tabatabaee A: Cadmium, nickel and vanadium accumulation by three strains of marine bacteria. Ira J Biotechnol 2006, 4:180-187.

4. Mabbett AN, Lloyd JR, Macaskie LE: Effect of complexing agents on reduction of $\mathrm{Cr}(\mathrm{VI})$ by Desulfovibrio vulgaris ATCC 29579. Biotechnol Bioeng 2002, 79:389-397.

5. Cheung K, Ji-Dong G: Chromate reduction by Bacillus megaterium TKW3 isolated from marine sediments. World J Microbiol Biotechnol 2005, 21:213-219.

6. Strickler DJ: Biomaterials to prevent nosocomial infections: is silver the gold standard? Curr Opin Infect Dis 2000, 13:389-393.

7. Percival SL, Bowler PG, Russell D: Bacterial resistance to silver in wound care. J Hospital Infection 2005, 60:1-7.

8. Cohen MS, Stern JM, Vanni AJ, Kelley RS, Baumgart E, Field D, Libertino JA, Summerhayes IC: In vitro analysis of a nanocrystalline silver-coated surgical mesh. Surg Infec 2007, 8:397-404.

9. Sondi I, Sondi BS: Silver nanoparticles as antimicrobial agent: a case study on E. coli as a model for Gram-negative bacteria. J Colloids Interface Science 2004, 275:177-182.

10. Mullen MD, Wolf DC, Ferris FG, Beveridge TJ, Flemming CA, Bailey GW: Bacterial sorption of heavy metals. Appl Environ Microbiol 1989, 55:3143-3149.

11. Beveridge TJ, Forsberg CW, Doyle RJ: Major sites of metal binding in Bacillus licheniformis walls. J Bacteriol 1982, 150:1438-1448.

12. Kalishwaralal K, Ramkumarpandia SB, Deepak V, Mohammad B, Sangiliyandi G: Biosynthesis of silver nanocrystals by Bacillus licheniformis. Coll surf B: Biointerface 2008, 65:153.

13. Ganesh Babu MM, Gunasekaran P: Production and structural characterization of silver nanoparticles from Bacillus cereus PGN1 isolate. Coll surf B: Biointerface 2009, 74:191-194.

14. Beveridge TJ, Murray RGE: Sites of metal deposition in the cell wall of Bacillus subtilis. J Bacteriol 1980, 141:876-887.

15. Anuradha P, Seema S, Naheed A, Ashok G, Preety S: Synthesis of AgNps By Bacillus cereus bacteria and their antimicrobial potential. J Biomaterial Nanobiotech 2011, 2:155-161.

16. Wilks JC, Kitko RD, Cleeton SH, Lee GE, Ugwu CS, Jones BD, BonDurant SS, Slonczewski JL: Acid and base stress and transcriptomic responses in Bacillus subtilis. Appl Environ Microbiol 2009, 75:981-990.

17. Helmann JD, Wu MFW, Gaballa A, Kobel PA, Morshedi MM, Fawcett P, Paddon C: The global transcriptional response of Bacillus subtilis to peroxide stress is coordinated by three transcription factors. J Bacteriol 2003, 185:243-253.

18. den Besten HMW, Mols M, Moezelaar R, Zwietering MH, Abee T: Phenotypic and transcriptomic analyses of mildly and severely saltstressed Bacillus cereus ATCC 14579 cells. Appl Environ Microbiol 2009, 75:4111-4119.

19. Steil L, Hoffmann T, Budde I, Volker U, Bremer E: Genome-wide transcriptional profiling analysis of adaptation of Bacillus subtilis to high salinity. J Bacteriol 2003, 185:6358-6370.

20. Mols M, Kranenburg RV, Tempelaars MH, Schaik W, Moezelaar R, Abee T: Comparative analysis of transcriptional and physiological responses of Bacillus cereus to organic and inorganic acid shocks. Int J Food Microbiol 2010, 137:13-21. 
21. Moore CM, Gaballa A, Hui M, Ye RW, Helmann JD: Genetic and physiological responses of Bacillus subtilis to metal ion stress. Mol Microbiol 2005, 57:27-40.

22. Passalacqua KD, Bergman NH, Lee JY, Sherman DH, Hanna PC: The global transcriptional responses of Bacillus anthracis strain $34 \mathrm{~F}_{2}$ and a sodA1 mutant to paraquat reveal metal ion homeostasis imbalances during endogenous superoxide stress. J Bacteriol 2007, 189:3996-4013.

23. Kristoffersen SM, Ravnum S, Tourasse NJ, Okstad OA, Kolsto AB, Davies W: Low concentration of bile salts induce stress responses and reduce motility in Bacillus cereus ATCC 14570. J Bacteriol 2007, 189:5302-5313.

24. Ivanova N, Sorokin A, Anderson I, Galleron N, Candelon B, Kapatral V, Bhattacharyya A, Reznik G, Mikhailova N, Lapidus A, Chu L, Mazur M, Goltsman E, Larsen N, D'Souza M, Walunas T, Grechkin Y, Pusch G, Haselkorn R, Fonstein M, Ehrlich SD, Overbeek R, Kyrpides N: Genome sequence of Bacillus cereus and comparative analysis with Bacillus anthracis. Nat 2003, 423:87-91.

25. Griffiths-Jones S, Moxon S, Marshall M, Khanna A, Eddy SR, Bateman A: Rfam: annotating non-coding RNAs in complete genomes. Nucl Acid Res 2005, 33:D121-D124.

26. Guzman MG, Dille J, Godet S: Synthesis of silver nanoparticles by chemical reduction method and their antibacterial activity. Int $J$ Che Bio Engg 2009, 2:104-111.

27. Benn $T M$, Westerhoff $P$ : Nanoparticle silver released into water from commercially available sock fabrics. Env Sci Technol 2008, 42:4133-4139.

28. Yue H, Eastman PS, Wang BB, Minor J, Doctolero MH, Nuttall RL, Stack R, Becker JW, Montgomery JR, Vainer M, Johnston R: An evaluation of the performance of CDNA microarrays for detecting changes in global mRNA expression. Nucl Acid Res 2001, 29:e41.

29. Solioz M, Odermatt A: Copper and silver transport by CopB-ATPase in membrain vesicles of Enterococcus hirae. J Biol Chem 1995, 270:9217-9221.

30. Prasad J, McJarrow P, Gopal P: Heat and osmotic stress responses of probiotic Lactobacillus rhamnosus HNO01 (DR20) in relation to viability after drying. Appl Environ Microbiol 2003, 69:917-925.

31. Periago PM, Van Schaik W, Abee T, Wouters JA: Identification of proteins involved in the heat stress response of Bacillus cereus ATCC 14579. App/ Environ Microbiol 2002, 68:3486-3495.

32. Hu P, Brodie EL, Suzuki Y, McAdams HH, Anderson GL: Whole-genome transcriptional analysis of heavy metal stresses in Caulobacter crescentus. J Bacteriol 2005, 187:8437-8449

33. Kumar SA, Abyaneh MK, Gosavi SW, Kulkarni SK, Pasricha R, Ahmad A, Khan MI: Nitrate reductase-mediated synthesis of silver nanoparticles from $\mathrm{AgNO}_{3}$. Biotechnol Lett 2007, 29:439-445.

34. Schaik WV, van der Voort M, Molenaar D, Moezelaar R, de Vos WM, Abee T: Idetification of the $\sigma^{B}$ regulon of Bacillus cereus and conservation of $\sigma^{B}$ regulated genes in low-GC-content Gram-positive bacteria. J Bacteriol 2007, 189:4384-4390.

35. Vido K, Spector D, Lagniel G, Lopez S, Toledano MB, Labarre J: A proteome analysis of the cadmium response in Saccharomyces cerevisiae. J Bio Chem 2001, 276:8469-8474.

36. Bae W, Chen X: Proteomic study for the cellular responses to $\mathrm{Cd}^{2+}$ in Schizosaccharomyces pombe through amino acid-coded mass tagging and liquid chromatography tandem mass spectrometry. Mol cell prot 2004, 3:596-607.

37. Trivedi VD, Spudich JL: Photostimulation of a sensory rhodopsin II/Htrll/ Tsr fusion chimera activates CheA-autophosphorylation and CheYphosphotransfer in vitro. Biochemistry 2003, 42:13887-13892.

38. Arnold JC, Sandrine K, Proux C, Fardeau ML, Dillies MA, Coppee JY, Ploetze FA, Bertin PN: Temporal transcriptomic response during arsenic stress in Herminiimonas arsenicoxydans. BMC Genomics 2010, 11:709-718.

39. Sridhar J, Narmada SR, Sabrinathan R, Ou HY, Deng Z, Sekar K, Rafi ZA, Rajakumar K: sRNAscanner: A computational tool for intergenic small RNA detection in bacterial genomes. Plos one 2010, 8:e11970.

doi:10.1186/1477-3155-9-49

Cite this article as: Ganesh Babu et al: Global transcriptome analysis of Bacillus cereus ATCC 14579 in response to silver nitrate stress. Journal of Nanobiotechnology 2011 9:49.

\section{Submit your next manuscript to BioMed Central and take full advantage of:}

- Convenient online submission

- Thorough peer review

- No space constraints or color figure charges

- Immediate publication on acceptance

- Inclusion in PubMed, CAS, Scopus and Google Scholar

- Research which is freely available for redistribution

Submit your manuscript at www.biomedcentral.com/submit
C Biomed Central 